University of Nebraska - Lincoln

DigitalCommons@University of Nebraska - Lincoln

Faculty Publications, Department of Mathematics

Mathematics, Department of

$11-3-1978$

An Illustrative Model Describing the Refraction of Long Water

Waves by a Circular Island

Gregory A. Kriegsmann

Follow this and additional works at: https://digitalcommons.unl.edu/mathfacpub

Part of the Applied Mathematics Commons, and the Mathematics Commons

This Article is brought to you for free and open access by the Mathematics, Department of at

DigitalCommons@University of Nebraska - Lincoln. It has been accepted for inclusion in Faculty Publications,

Department of Mathematics by an authorized administrator of DigitalCommons@University of Nebraska - Lincoln. 


\title{
An Illustrative Model Describing the Refraction of Long Water Waves by a Circular Island
}

\author{
Gregory A. KriegsmanN \\ Department of Mathematics and Statistics, University of Nebraska at Lincoln, Lincoln, NE 68588
}

(Manuscript received 28 August 1978, in final form 3 November 1978)

\begin{abstract}
The refraction of small shallow water waves by an idealized circular island is studied in this paper. The island's shoal is modeled by a quartic polynomial in the radial variable. This particular model allows the explicit construction of the rays (wave orthogonals) and the determination of several important features of the wave motion. The various shortcomings of the particular profile are discussed.
\end{abstract}

\section{Introduction}

In this paper we shall study the refractive properties of a circular island with a specific depth profile. The linear shallow water theory is assumed to be valid. This assumption requires that $h / \lambda \ll 1$, where $\lambda$ is the wavelength of the incident plane wave and $h$ the depth of the fluid. We shall apply the method of geometrical optics (Arthur, 1946) to obtain an approximate description of the wave motion in the short wave limit. This limit requires that $h / a \ll 1$, where $a$ is a typical horizontal dimension. Since this parameter must characterize the shoal's slope and the island's size, we choose it to be both the island's radius $\left(r_{0}\right)$ and $h /|\nabla h|$. The short wave limit is then given by the constraints $|\nabla h| \ll 1$ and $h / r_{0} \ll 1$. Thus we are concerned with the refractive properties of large islands with gently sloping shoals. We offer here no justification of this short wave approximation to the shallow water (long wave) equations. The reader is referred to Jonsson et al. (1976, 1978) where such matters are amply discussed and clarified. However, the method of geometrical optics does give a certain amount of qualitative in formation regarding the amplitude and phase of the wave motion. The description is elegant in its simplicity. Moreover, the rays play an important role in the qualitative study of the time-dependent wave equation (Lowell, 1948). Discontinuities and other singularities propagate along these curves.

The depth profile we use differs from those previously studied in the literature. Quadratic profiles, in the radial variable $r^{\prime}$, are often used to model the trapping of waves around the island; the wave orthogonals (rays) then become logarithmic spirals (Jonsson et al. 1976). Linear depth profiles in $r^{\prime}$ are sometimes used for experimental and computational reasons; the wave heights are bounded at the shoreline (Lautenbacher, 1970;
Smith and Sprinks, 1975). These models are respectively called "parabolic and conical" islands.

Our island has a depth profile which is quartic in $r^{\prime}$. This choice allows us to determine the rays explicitly: they are merely arcs of circles. It is interesting to note that this particular profile has been used in other mathematical and physical fields (Luneburg, 1964, Sec. 28).

In Section 2 of this paper we formulate the physical problem and briefly describe the method of geometrical optics. In Section 3 we calculate the rays and note several interesting features. Finally; in Section 4 we compute the amplitude and phase of the wave motion. We discuss the shortcomings of our method and model.

\section{Formulation}

An idealized circular island is attacked by a small, monochromatic plane wave. The island is situated in an infinite ocean of constant depth $H$. The depth of the water surrounding the island is given by

$$
h\left(r^{\prime}\right)= \begin{cases}H, & r^{\prime} \geqslant R \\ \frac{H\left(r^{\prime 2}-r_{0}^{2}\right)^{2}}{\left(R^{2}-r_{0}^{2}\right)^{2}}, & r_{0} \leqslant r^{\prime} \leqslant R\end{cases}
$$

where $r_{0}$ is the island's radius, $r^{\prime 2}=x^{\prime 2}+y^{\prime 2}$, and $R$ is the radius of the island's shelf (see Fig. 1). According to the shallow water theory (Lamb, 1945) the harmonic wave height $\eta e^{i \omega t}$ satisfies the reduced wave equation (scaled with respect to $r_{0}$ )

$$
\nabla^{2} \eta-\frac{\nabla \mu^{2} \cdot \nabla \eta}{\mu^{2}}+k^{2} \mu^{2} \eta=0,
$$

where $\mu^{2}=H / h\left(x^{\prime} / r_{0}, y^{\prime} / r_{0}\right), k^{2}=r_{0}^{2} \omega^{2} / C, C$ is the phase velocity $(g H), g$ the gravitational constant, and $\omega$ the frequency of the incident wave. The func- 


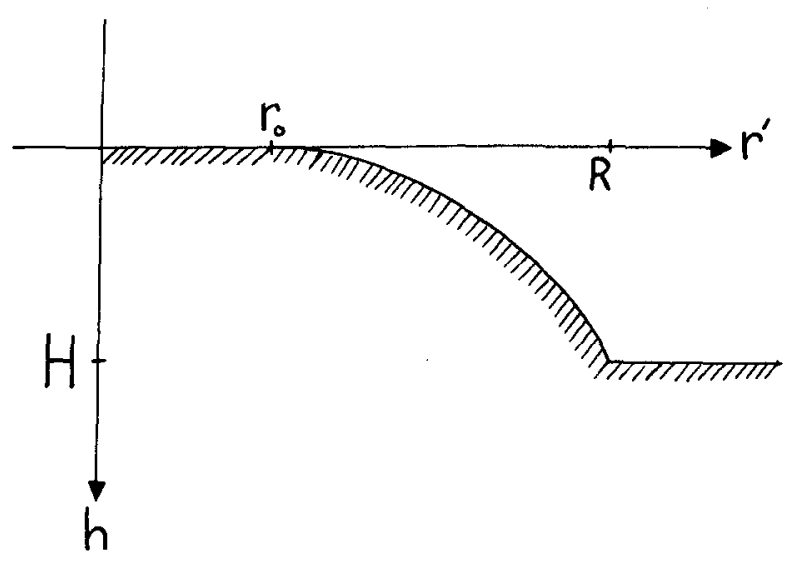

Fig. 1. The model depth profile.

tion $\mu^{2}$ is given in terms of the dimensionless variable, $r=r^{\prime} / r_{0}$, by

$$
\mu^{2}= \begin{cases}1, & r \geqslant d \\ \frac{\left(d^{2}-1\right)^{2}}{\left(r^{2}-1\right)^{2}}, & 1 \leqslant r \leqslant d\end{cases}
$$

where $d=R / r_{0}$.

In addition to satisfying (2.2) $\eta$ and $\nabla \eta$ must be continuous for $r \geqslant 1$. Moreover, the scattered wave must satisfy the radiation condition at infinity. That is, for $r \geqslant d$ we have

$$
\eta=e^{i k x}+\eta_{s}
$$

where the scattered wave $\eta_{s}$ satisfies

$$
\eta_{s} \sim f(\theta) \frac{e^{i k r}}{r^{1 / 2}} \text { as } r \rightarrow \infty .
$$

The total wave $\eta$ has been made dimensionless with respect to the amplitude of the incident wave. The exponential term in (2.4a) represents the incident plane wave which is progressing in the direction of the negative $x$ axis.

We shall now suppose that $k$ is "large" and seek a solution of the form

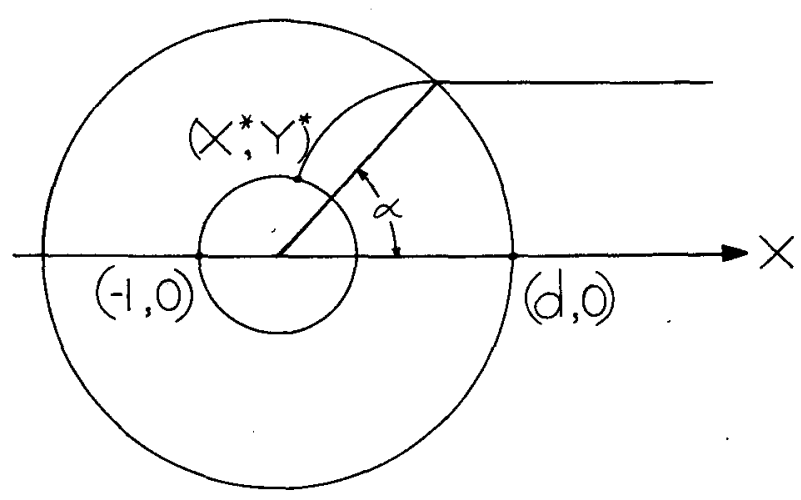

Fig. 2. A typical ray.

$$
\eta(x, y ; k)=e^{i k \psi(x, y)}[A(x, y)+\mathrm{O}(1 / k)]
$$

as $k \rightarrow \infty$. The term $\mathrm{O}(1 / k)$ is a function of $x, y$ and $k$ which is bounded by $k^{-1}$ as $k \rightarrow \infty$. Eq. (2.5) is the "ansatz" of geometrical optics and is assumed to be valid except in certain regions where other expansions are appropriate. Thus, our first task is to compute $A$ and $\psi$ and determine where (2.5) breaks down. Substituting (2.5) and (2.3) into (2.2) and equating the coefficients of the powers of $k$ to zero, we obtain

$$
\nabla \psi \cdot \nabla \psi=\mu^{2} \quad \text { (eikonal equation), }
$$

$2 \nabla A \cdot \nabla \psi+A \nabla^{2} \psi$

$$
-A \frac{\nabla \mu^{2} \cdot \nabla \psi}{\mu^{2}}=0 \text { (transport equation). }
$$

\section{The rays}

The eikonal equation is a nonlinear, first-order, partial differential equation. We will solve it by introducing a one-parameter family of curves called "rays" in the optics literature (or "wave orthogonals" in the marine literature) which are everywhere perpendicular to the curves $\psi=$ constant (Arthur, 1946; Synge, 1962). Since the incident wave is plane, its rays are horizontal lines. We consider the incident ray which strikes the island's shelf at $(d \cos \alpha, d \sin \alpha$ ) (see Fig. 2). It is convenient to use $\alpha$ as the parameter which labels a ray. If we introduce the parametric representation of the ray, $\mathbf{X}(s ; \alpha)$, where $s$ is the arclength, the orthogonality condition is

$$
\frac{D \mathbf{X}}{D s}=\frac{\nabla \psi}{\mu} .
$$

Moreover, by differentiating this equation and using Eq. (2.6), we find that $X$ satisfies

$$
\frac{D}{D s}\left(\mu \frac{D \mathbf{X}}{D s}\right)=\nabla \mu \text {. }
$$

This differential equation with $\mu^{2}$ given by (2.3) arises in other physical and mathematical situations. For example, it gives the path of light in Maxwell's model of a fish eye (Luneburg, 1964, Sec. 28). Luneburg gives an elegant solution of the differential equation by applying certain techniques and results of differential geometry; he finds that the rays are circles. In the next few paragraphs we give an elementary proof of this result.

Let $s=0$ correspond to the point where the incident ray strikes the island's shelf. The initial data needed to solve (3.2) are deduced from the definition of $\alpha$ [Eq. (3.1)], the form of the incident wave and the continuity of $\eta$. We find that 
$\left.\begin{array}{l}\mathbf{X}(0 ; \alpha)=d(\cos \alpha, \sin \alpha),|\alpha| \leqslant \frac{\pi}{2} \\ \frac{D \mathbf{X}}{D s}(0 ; \alpha)=(-1,0) \quad \text { and } \quad \psi(0 ; \alpha)=\cos \alpha\end{array}\right\}$

Let $\mathrm{V}_{1}$ and $\mathrm{L}$ be defined by

$$
\begin{aligned}
\mathbf{V}_{1} & =\frac{D \mathbf{X}}{D s}, \\
\mathbf{L} & =\mathbf{X} \times \mu \mathbf{V}_{1} .
\end{aligned}
$$

One is tempted here to draw an analogy between a ray and the trajectory of a particle in a conservative force field. Indeed, $\mu V_{1}$ can be interoreted as the velocity of the particle and $\mu$ as the potential. Then (3.2) becomes the statement of Newton's second law. In this spirit, $\mathrm{L}$ is the angular momentum and is conserved along the trajectory. This fact readily follows from (3.2), (3.4), and the radial symmetry of $\mu$.

The curvature of a ray $K$ is defined by

$$
\frac{D \mathbf{V}_{1}}{D s}=K \mathbf{V}_{2} \text {, }
$$

where $V_{2}$ is the unit normal. By taking the scalar product of (3.2) with $\mathbf{V}_{2}$ it follows that

$$
K=\frac{1}{\mu} \nabla \mu \cdot \mathbf{V}_{2} \text {. }
$$

Next, we let $r-\phi$ be the angle between $V_{1}$ and $\mathbf{X}$ (see Fig. 3). Since $\nabla \mu=r^{-1} \mathbf{X}(\partial \mu / \partial r),(3.4)$ and (3.6) give

$$
\begin{aligned}
K & =\frac{-1}{r} \frac{\partial \mu}{\partial r} \sin \phi, \\
|\mathrm{L}| & =r \mu \sin \phi .
\end{aligned}
$$

From (3.3) and (3.4) and the fact that $L$ is co.nstant along a ray, it follows from combining (3.7) and (3.8) that

$$
\frac{\mu_{r}}{\mu^{2}}=\frac{-r K}{d \sin \alpha} .
$$

This result is valid for any radially symmetric depth profile.

Inserting our particular model $\mu^{2}$ [Eq. (2.3)] into (3.9) yields the result ${ }^{1}$

$$
K=2 d \sin \alpha /\left(d^{2}-1\right),
$$

$f_{\mathrm{C}} \mathrm{z} \leqslant r \leqslant d$. Thus, for a given $\alpha$, the ray is $\varepsilon$.n arc of a circle with radius $\rho=1 / K$ and center

$$
\left(x_{c}, y_{c}\right)=(d \cos \alpha, d \sin \alpha-\rho) \text {. }
$$

The equation of a ray is

\footnotetext{
${ }^{1}$ The converse is also true: if $K$ is constant, then (3.9) yields $\mu=\left(C_{1} r^{2}+C_{2}\right)^{-1}$ for arbitrary constants $C_{1}$ and $C_{2}$.
}

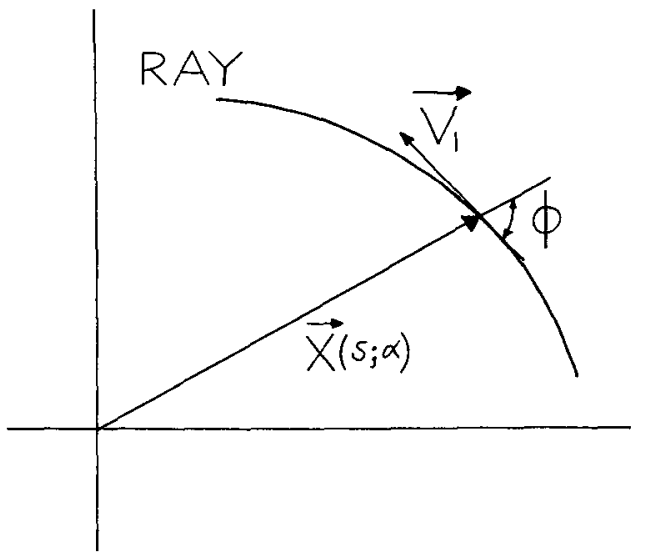

Fig. 3. The tangent vector and the angle $\phi$.

$$
\left.\begin{array}{l}
x=x_{c}-\rho \sin K s \\
y=y_{c}+\rho \cos K s
\end{array}\right\} .
$$

These wave orthogonals possess two interesting features. First, each ray strikes the shore perpendicularly. This follows from (3.8): since $|L|$ is a constant along a ray and $\mu \rightarrow \infty$ as $r \rightarrow 1$, then $\sin \phi \rightarrow 0$ or $\phi \rightarrow 0$. Second, a straightforward trigonometric calculation shows that the ray strikes the island at

$$
\left(x^{*}, y^{*}\right)=\left(1+\rho^{2}\right)^{-1}\left(x_{c}-\rho y_{c}, \rho x_{c}+y_{c}\right) .
$$

From this result it follows that some rays end up on the leeward side of the island. In particular as $\alpha \rightarrow \pi / 2$, the limiting ray strikes the shore at

$$
(\tilde{x}, \bar{y})=\left(1+d^{2}\right)^{-1}\left(1-d^{2}, 2 d\right) .
$$

In Fig. 4 we have sketched several rays and wavefronts $(\psi=$ constant). It is apparent from this figure that the wave, as it penetrates into the shallow water, "feels" the bottom and "wraps" itself around the island. Finally, it should be noted that a region, devoid of rays, exists on the leeward side of the island. This is the geometrical shadow.

\section{The phase and amplitude}

Once the rays have been determined, the phase function may be readily deduced. From (2.6) and

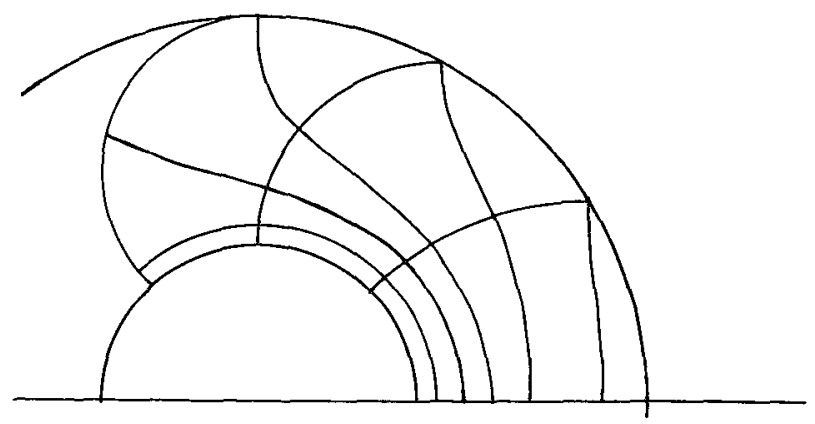

FIG. 4. Several rays and wave fronts. 


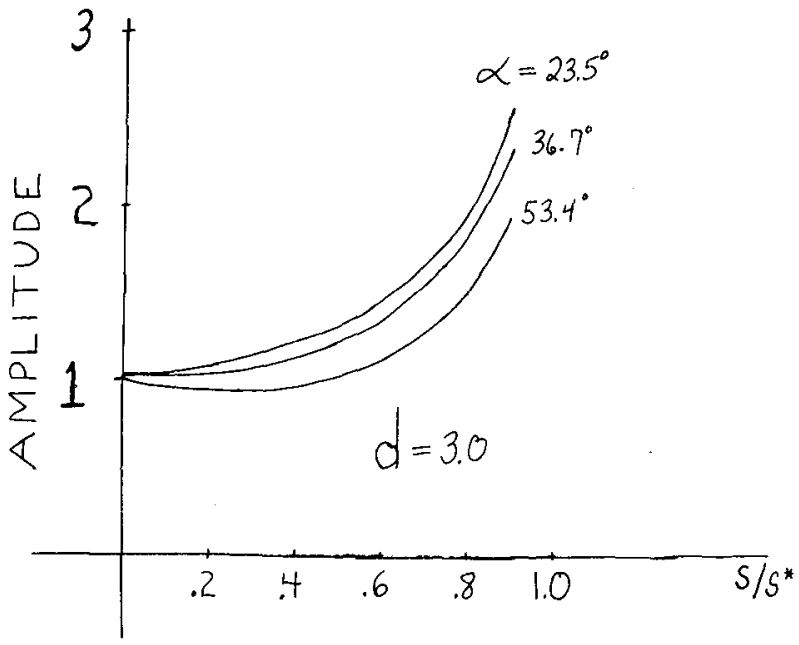

FIG. 5. The amplitude along various rays.

(3.1) it follows that $\psi$ satisfies the differential equation

$$
\frac{D \psi}{D s}=\mu
$$

along a ray. From the initial conditions (3.3) and (3.4) we find that

$$
\psi=\cos \alpha+\int_{0}^{s} \mu\left(\left|\mathbf{X}\left(s^{\prime} ; \alpha\right)\right|\right) d s^{\prime} .
$$

The inversion of (3.12) would give $s$ and $\alpha$ as functions of $x$ and $y$. When these expressions are substituted into (4.2), the phase function is determined for each point $(x, y)$ in the region covered by the rays. Unfortunately, this procedure is difficult to carry out even though the rays are simple. ${ }^{2}$ The inversion can be carried out, in principle as long as the Jacobian

$$
\left(J=\frac{\partial x}{\partial s} \cdot \frac{\partial y}{\partial \alpha}-\frac{\partial y}{\partial s} \cdot \frac{\partial x}{\partial \alpha}\right)
$$

does not vanish. From (3.12) it follows that

$$
J=-d \cos (K s-\alpha)+\rho \cot \alpha(1-\cos K s) \text {. }
$$

A long and involved calculation shows that $J<0$. We do not produce it here. However, from Fig. 4 it appears that only one ray passes through each point in the "irradiated" region. This fact is sufficient to guarantee the inversion of (3.12) and imply $J \neq 0$.

Although the inversion appears impossible to analytically perform, we can still deduce an important qualitative feature of $\psi$. Let $s^{*}$ correspond to the arclength of a ray when it strikes the shoreline. At this point $r=1$ and $\mu$ becomes infinite. A

\footnotetext{
${ }^{2}$ The functional dependence of $\alpha$ on $x$ and $y$ is obtained by
} finding the roots of a quartic in $\sin \alpha$. careful analysis of the integral reveals that $\psi$ is logarithmically singular at the shoreline. Thus the period of the wave approaches zero as $r \rightarrow 1$. This potential problem is a shortcoming of our depth profile and not the geometrical optics method. Indeed, if $h(r)$ were linear in $r$, the singularity in (4.2) would be integrable and $\psi$ would be bounded at the shoreline.

We shall now describe an interesting physical observation which follows from the form of $\psi$. From (3.8) it follows that the rays perpendicularly strike the shoreline for any radially symmetric $\mu(r)$ which becomes unbounded as $r \rightarrow 1$ (or equivalently, $h \rightarrow 0$ at the shoreline). Consider a portion of the incident plane wave given by $x=x_{0}>d$ and $|y|$ $\leqslant d$. The wave orthogonals are horizontal lines until they reach the island's shelf $(r=d)$ where they bend and perpendicularly strike the shoreline. Thus, the shoreline appears to be a wave front where $\psi$ $=$ constant. For our particular model $\psi=\infty$ while for others the constant is bounded. Now, from (4.1) we have that the integral $\int \mu d s$ is invariant along all the rays joining the wave fronts $\psi(r=1)$ and $\psi\left(x=x_{0}\right)$. Since this integral is physically proportional to time, all the wave crests (troughs) initially at $x=x_{0}$ will strike the island at the same time. For our particular model the waves "pile up" and never reach the shoreline while for other models they do so in a finite amount of time.

Before computing the amplitude we find it convenient to rewrite the transport equation (2.7) in the divergence form

$$
\nabla \cdot\left(\frac{A^{2}}{\mu^{2}} \nabla \psi\right)=0 .
$$

By integrating this expression over a small bundle of rays, applying the divergence theorem, and changing variables, we show in the Appendix that

$$
A=\left(\frac{\mu d \cos \alpha}{|J|}\right)^{1 / 2},
$$

where $J$ is given by (4.3). This result is valid for any radially symmetric depth profile. Now as $s \rightarrow s^{*}, r \rightarrow 1$ and $A$ becomes unbounded. This singularity is a result of the asymptotic method and not the particular depth profile. It suggests that an expansion, different from the geometrical optics ansatz, is needed near the shoreline. Moreover, there is a possibility that the nonlinear equations are required as $r \rightarrow 1$. Finally, in Fig. 5 we show $A$ as a function of $s / s^{*}$ along several rays.

Acknowledgment. The author wishes to thank Professor R. E. Meyer for his kind encouragement in the preparation of this manuscript and Professor I. G. Jonsson for his many helpful comments and suggestions. 


\section{APPENDIX}

\section{Derivation of Eq. (4.5)}

We consider two neighboring rays, $\mathbf{X}(s ; \alpha)$ and $\mathbf{X}(s ; \alpha+\delta \alpha)$, where $|\delta \alpha| \ll 1$. For $s$ fixed let $C_{s}$ $=\{(x, y) \mid(x, y)=\mathbf{X}(s ; u), \alpha \leqslant u \leqslant \alpha+\delta \alpha\}$. Then $C_{0}$ is the arc of the island's shelf in between the two rays. Let $\delta l_{s}$ represent the length of $C_{s}$ and assume $\delta l_{s} \leqslant 1$. Let $D_{\delta}$ be the region bounded by the neighboring rays, $C_{0}$ and $C_{s}$. From (4.4) it follows that

$$
0=\iint_{D_{\delta}} \nabla\left(\frac{A^{2}}{\mu^{2}} \nabla \psi\right) d x d y .
$$

By applying the divergence theorem (more precisely, Green's theorem) to this result and using (3.1) one finds that

$$
\cos \beta_{s} \frac{A^{2}}{\mu} \delta l_{s} \approx \cos \beta_{0} \frac{A_{0}^{2}}{\mu_{0}} \delta l_{0},
$$

where the right-hand side is evaluated at a point on $C_{0}$, the left-hand side is evaluated at a point on $C_{s}$, and $\beta_{s}$ is the angle between the normal to $C_{s}$ and $\nabla \psi$. Since the energy $\sim A^{2}$ and the group speed equals the phase velocity $\left[(g h)^{1 / 2} \approx 1 / \mu\right]$, Eq. (A.2) simply states that the mean energy flux is constant between neighboring orthogonals. Now, it is easy to verify that

$$
\cos \beta_{s} \delta l_{s}=J_{s} \delta \alpha,
$$

where $J_{s}$ is the Jacobian Eq. (4.3) evaluated at a point on $C_{s}$. As $\delta \alpha$ approaches zero, $\delta l_{s} \rightarrow 0$, $\delta l_{0} \rightarrow 0$ and (A.2) becomes an identity. Noting that $A_{0}=\mu_{0}=1$ and $\left|J_{0}\right|=d \cos \alpha$, it follows from (A.2) that

$$
A=\left(\frac{d \mu \cos \alpha}{|J|}\right)^{1 / 2}
$$

\section{REFERENCES}

Arthur, R. S., 1946. Refraction of water waves by islands and shoals with circular bottom contours. Trans. Amer. Geophys. Union., 27, 168-177.

Jonsson, I. G., O. Skovgaard and O. Brink-Kjaer, 1976. Diffraction and refraction calculations for waves incident on an island. J. Mar. Res., 34, 469-496.

$\ldots$, and —, 1978. A mild-slope wave equation and its application to tsunami calculations. Mar. Geod., 2, 41-58.

Lamb, H., 1945. Hydrodynamics. New York, Dover, 250-358.

Lautenbacher, C. C., 1970. Gravity wave refraction by islands. J. Fluid Mech., 41, 655-672.

Lowell, S. C., 1948. The propagation of waves in shallow water. Commun. Pure Appl. Math., 2, 275-291.

Luneburg, R. K., 1964. Mathematical Theory of Optics. Berkeley, University of California Press (see pp. 172-178).

Smith, R, and T. Sprinks, 1975. Scattering of surface waves by a conical island. $J$. Fluid Mech., 72, 373-384.

Synge, J. L., 1962. Geometrical Optics. Cambridge University Press, 99-110. 